\title{
Fetal Alcohol Syndrome: Do Vitamins Have A Role?
}

\section{Michael N. A. Eskin*}

Department of Human Nutritional Sciences, University of Manitoba, Canada

Fetal Alcohol Syndrome (FASD) is a condition associated with alcohol consumption during pregnancy that results in dysmorphic facial features, impaired growth and central nervous system abnormalities [1]. The degree of damage depends on the extent and regularity of alcohol consumption during pregnancy, and is particularly prevalent in individuals of low socioeconomic status. In addition to vitamin $\mathrm{A}$ deficiency, those living at the poverty level may also be deficient in folate and choline, both of which are important for normal neural tube development. This editorial will focus on the potential of these vitamins in mitigating some of the effects of alcohol in the development of FASD.

Depletion of vitamin A stores, as a result of alcohol consumption, can lead to growth retardation, limb dysmorphogenesis, as well dysfunction of the central nervous system [2]. This is due to the direct competition between vitamin A or retinol and alcohol for the enzyme alcohol dehydrogenase, which has a higher affinity for binding to alcohol. As a result, vitamin A metabolism is blocked, causing a deficiency in retinoic acid synthesis [2,3]. Retinoic acid is essential for fetal development, organogenesis, organ homeostasis, cell and neuronal growth and differentiation, development of the central nervous system, as well as limb morphogenesis [2,4]. By exposing Xenopus laevis frog embryos to ethanol, Yelin et al. [5] found a high incidence of malformations that resembled the phenotypic anomalies induced in individuals affected by FASD. Further work by Yelin et al. [6] on early molecular changes induced by ethanol exposure established the suitability of this model for studying FASD. Later work by using Zebrafish embryos as their model, confirmed the defects in retinoic acid signalling, resulting from embryonic exposure to alcohol. Supplementation of ethanol exposed Zebra fish embryos with low levels of retinoic acid $\left(10^{-9} \mathrm{M}\right)$, during grastrulation and somitogenesis, significantly restored some of the defects caused by alcohol exposure. However, fetal anomalies reported by excess consumption of retinoic acid, suggests there is only a very narrow safe range for this nutrient [7].

Folic acid, an essential nutrient that plays a key role in one-carbon transfers, is intimately involved in the biosynthesis of DNA and DNA methylation [8]. During pregnancy, there is an increased demand for folic acid to support the rapid growth of the fetus. However, ethanol exposure can severely impair folate by altering absorption, distribution and excretion [9-11]. Hewitt et al. [12] examined the effects of chronic ethanol exposure and folic acid supplementation on the folic acid status of a maternal and fetal guinea pig model. While folate supplementation did not alleviate the decrease of folic acid in the brain and hippocampus, it did prevent a decrease in hepatic levels, in both the mother and fetus. Previous work by Cano et al. [13] also found that folate supplementation had a protective effect by reducing oxidative stress in the offspring of pregnant rats exposed to ethanol [14]. Later reported that a high folate diet prevented ethanol-induced cardiac defects in pregnant mice exposed to a intraperitoneal injection, equivalent to a binge-drinking dose of ethanol.

Choline is another essential nutrient required for fetal development [15]. Addition of choline during early postnatal development was reported by Thomas et al. [16], to reduce the severity of memory deficits induced in adult rats subjected to prenatal alcohol exposure. Supplementation of adult rats with choline from postnatal days, 4-30 days was also found to reduce the severity of a number of behaviour symptoms associated with ethanol exposure, such as spatial learning deficits and over activity [17], trace eye blinking conditioning [18], and trace fear conditioning deficits [19]. Such changes were associated with alcohol exposure during the third trimester equivalent brain growth spurt [20]. Choline supplementation, coincident with prenatal alcohol exposure in rats, was also shown by Thomas et al. [20] to protect against ethanol's terratogenic effects.

All these studies point to vitamin A, folate and choline mitigating some of the adverse effects of ethanol exposure, consistent with that presented recently by Ballard et al. [21]. Future studies, including epigenetics, will shed more light on the potential protective and preventive roles of these and other vitamins in reducing the abnormalities associated with the development of fetal alcohol syndrome.

\section{References}

1. Jones KL, Smith DW (1973) Recognition of the fetal alcohol syndrome in early infancy. Lancet 302: 999-1001.

2. West JR, Chen WJ, Pantazis NJ (1994) Fetal alcohol syndrome: The vulnerability of the developing brain and possible mechanisms of damage. Metab Brain Dis 9: 291-322.

3. Marrs JA, Clendenson SG, Ratcliffe DR, Fielding SM, Liu Q, et al. (2010) Zebrafish fetal alcohol syndrome model: effects of ethanol are rescued by retinoic acid supplement. Alcohol 44: 707-715

4. Kumar A, Singh CK, DiPette DD, Singh US (2010) Ethanol impairs activation of retinoic acid receptors in cerebellar granule cells in a rodent model of fetal alcohol spectrum disorders. Alcohol Clin Exp Res 34: 928-937.

5. Yelin R, Schyr RB, Kot H, Zins S, Frumkin A, et al. (2005) Ethanol exposure affects gene expression in the embryonic organizer and reduces retinoic acid levels. Dev Biol 279: 193-204.

6. Yelin R, Kot H, Yelin D, Fainsod A (2007) Early molecular effects of ethano during vertebrate embryogenesis. Differentiaton 75: 393-403.

7. Zachman RD, Grummer MA (1998) The interaction of ethanol and vitamin A as a potential mechanism for the pathogenesis of fetal alcohol syndrome. Alcohol Clin Exp Res 22: 1544-1556.

8. Bailey LB, Gregory JF 3rd (1999) Folate metabolism and requirements. J Nutr 129: 779-782.

9. Hamid A, Kaur J, Mahmood A (2007) Evaluation of the kinetic properties of the folate transport system in intestinal absorptive epithelium during experimental ethanol ingestion. Mol Cell Biochem 304: 265-271.

10. Tamura T, Halsted CH (1983) Folate turnover in chronically alcoholic monkeys J Lab Clin Med 101: 623-628.

11. Muldoon RT, McMartin KE (1994) Ethanol acutely impairs the renal conservation of 5-methyltetrahydrofolate in the isolated perfused rat kidney. Alcohol Clin Exp Res 18: 333-339.

${ }^{*}$ Corresponding author: Michael N.A. Eskin, Professor, Department of Human Nutritional Sciences, University of Manitoba, Canada, E-mail: Michael.Eskin@ad.umanitoba.ca

Received February 15, 2013; Accepted February 17, 2013; Published February 24,2013

Citation: Eskin MNA (2013) Fetal Alcohol Syndrome: Do Vitamins Have A Role? Vitam Trace Elem 2: e121.

Copyright: (c) 2013 Eskin MNA. This is an open-access article distributed under the terms of the Creative Commons Attribution License, which permits unrestricted use, distribution, and reproduction in any medium, provided the original author and source are credited. 
12. Hewitt AJ, Knuff AL, Jefkins MJ, Collier CP, Reynolds JN, et al. (2011) Chronic ethanol exposure and folic acid supplementation: Fetal growth and folate status in the maternal and fetal guinea pig. Reprod Toxicol 31: 500-506.

13. Cano MJ, Ayala A, Murillo ML, Carreras O (2001) Protective effect of folic acid against oxidative stress produced in 21-day postpartum rats by maternalethanol chronic consumption during pregnancy and lactation period. Free Radic Res 34: 1-8

14. Serrano M, Han M, Brinez P, Linask KK (2010) Fetal alcohol syndrome: cadiac birth defects in mice and prevention with folate. Am J Obstet Gynecol 203: 75.

15. Zeisel SH (2006) Choline: critical role during fetal development and dietary requirements in adults. Annu Rev Nutr 26: 229-250.

16. Thomas JD, La Fiette MH, Quinn VR, Riley EP (2000) Neonatal choline supplementation ameliorates the effects of prenatal alcohol exposure on a discrimination learning task in rats. Neurotoxicol Teratol 22: 703-711.
17. Thomas JD, Garcia GG, Dominguez HD, Riley EP (2004) Administration of eliprodil during ethanol withdrawal in the neonatal rat attenuates ethanolinduced learning deficits. Psychopharmacology (Berl) 175: 189-195.

18. Tran T, Thomas JD (2007) Prenatal choline supplementation mitigates trace eyeblik deficits associated with $3^{\text {rd }}$ trimester alcohol exposure in rats. Society for Neuroscience.

19. Wagner AF, Hunt PS (2006) Impaired trace fear conditioning following neonata ethanol: reversal by choline. Behav Neurosci 120: 482-487.

20. Thomas JD, Abou EJ, Dominguez HD (2009) Prenatal choline supplemenmtation mitigates the adverse effects of prenatal alcohol exposure on development in rats. Neurotoxicol Teratol 31: 303-311.

21. Ballard MS, Sun M, Ko J (2012) Vitamin A, folate, and choline as a possible preventive intervention to fetal alcohol syndrome. Med Hypotheses 78: 489493. 